\title{
Special Issue: Wireless Sensor and Actuator Networks for Smart Cities
}

\author{
Burak Kantarci ${ }^{1, *,+}$ (D) and Sema F. Oktug $2, *,+\mathbb{D}$ \\ 1 School of Electrical Engineering and Computer Science, University Ottawa, Ottawa, ON K1N 6N5, Canada \\ 2 Department of Computer Engineering, Faculty of Computer and Informatics Engineering, Istanbul \\ Technical University, 34469 Istanbul, Turkey \\ * Correspondence: burak.kantarci@uottawa.ca (B.K.); oktug@itu.edu.tr (S.F.O.); \\ Tel.: +1-613-562-5800 (ext. 6955) (B.K.); +90-212-285-3584 (S.F.O.) \\ + All authors contributed equally to this work.
}

Received: 14 November 2018; Accepted: 15 November 2018; Published: 17 November 2018

\section{Introduction}

Our lives are being transformed by the interplay between mobile networks, wireless communications, and artificial intelligence. This transformation is an outcome of the emerging Internet of Things (IoT) concept, and the advancements in computer architectures that translate into high computing power, high-performance processing, and huge memory capacities. In addition to the IoT, as a very close concept, cyber-physical systems target seamless integration of physical systems with computing and communication resources. Furthermore, in urban areas, the integration of the "software-defined sensor networks" and "sensing as a service" concepts with legacy Wireless Sensor Network (WSN)-based systems is leading to the transformation of conventional city services towards smart cities.

Smart energy, smart driving, smart homes, smart living, smart governance, and smart health are just a few services that can be offered by smart cities. Furthermore, while these concepts are major application areas, smart citizens close the loop by participating in sensing, actuating, and decision-making processes. In smart cities, legacy WSN-based services are extended by having citizens that act as sensors. Opportunistic or participatory sensing models enable groups of individuals to collaboratively work toward the same goal with strong interaction links, even though this does not always require strong social links between them. Thus, dedicated and nondedicated wireless sensors form communities, and collaborating communities form social networks where interaction can occur in the form of software-defined sensing. This transformation in WSNs introduces unique solutions for the communication plane of smart cities.

In addition to communication-plane challenges, smart environments require IoT and WSN sensors to report massive amounts of unstructured data in a heterogeneous format, which, in turn, leads to the big sensed data phenomenon. Additionally, addressing the high volume by effective machine-learning or data-mining techniques, novel data-acquisition and -processing methodologies for big sensed data are emergent in order to address the high-velocity, -variety, and -veracity aspects. Moreover, in order to effectively offer smart-city services, it is viable to envision a massive amount of connected wireless/wired sensors/IoT devices. Thus, scalability remains an open issue when integrating the components of a smart city that are mentioned above. While ensuring the scalability and connectivity of this infrastructure remains an open issue, the battery limitation of wireless sensors is a great challenge, especially in time-sensitive services in smart cities.

In this Special Issue, we sought contributions that focus on novel solutions for Wireless Sensor and Actuator Networks (WSANs) in smart cities. The Special Issue has had contributions from academic and industry researchers in computer science and engineering, electrical engineering and 
communication engineering, as well as ICT industry engineers and practitioners. The contributions were original articles in all aspects of wireless sensor networks and actuator systems for smart cities. Particular topics of interest were as follows:

- Physical layer challenges in WSNs in smart-city applications.

- Cross-layer solutions for WSNs and IoT to support smart-city services.

- WSN and IoT architectures, protocols, platforms, and algorithms.

- Device-to-device networks for smart cities.

- Application-layer protocols for WSNs to enable efficient smart-city applications.

- Planning of sensor networks in smart cities,

- The interplay between dedicated and nondedicated sensing.

- Opportunistic and participatory sensing in smart cities.

- Design and Management of Mobile Crowd-Sensing Systems in smart cities.

- Energy-harvesting solutions for WSNs in smart cities.

- Vehicular sensing solutions for smart-city applications.

- Novel sensory data-acquisition techniques.

- Real-time and near-real-time data analytics on sensory data,

- Software-defined sensor networks and sensing as a service in smart cities.

- Security, privacy, and trust in smart-city sensing.

- Smart-city big data and open data.

- Standards for IoT and WSNs in smart-city applications.

- Application, deployment, testbed, experimental experiences, and innovative applications for WSN-enabled smart cities.

- IoT-driven smart governance, smart economy, and smart environments.

The Special Issue has covered most of these research topics by an outstanding collection of featured articles that have been selected through a rigorous peer-review process. The accepted articles have introduced further investigations beyond the listed topics under the smart-cities context. The contributions of the articles in this Special Issue are summarized in the following section.

\section{Summary of Contributions}

This special issue is a collection of unique contributions that address various issues in WSANs and smart cities by providing useful insights for future research in this field. After a rigorous and iterative peer-review process, eight papers have been selected by considering recommendations and feedbacks of at least three independent reviewers per paper in at least two rounds of review. The papers in this Special Issue have been contributed by 27 authors from academia and industries spanning various regions in the world, particularly North America, Europe, Asia, and North Africa. Each paper cites high-impact and scholarly references in the literature that make up a pool of 251 state-of-the-art references in total for further investigation in the research topics.

The articles that appear in this Special Issue form a diverse collection of topics studied under the scope of WSANs for smart cities. These include low-cost IoT implementation for smart-village settings [1], smart parking systems exploiting WSNs [2], Machine-To-Machine (M2M) Networking over LTE for smart- city services [3], WSN-driven smart waste-management systems for sustainable cities [4], user-support systems with wearable sensors and cameras [5], a SmartInsoles Cyber-Physical System (CPS) for mobile gait analysis [6], energy-harvesting systems for WSNs [7], and IoT for WSNs in smart cities [8].

Smart villages are promising infrastructures under the smart-cities concept. The selection of proper wireless access technologies for smart villages is of paramount importance. The author of Reference [1] presents a smart-village setting and proposes a conceptual framework to evaluate the cost of IoT deployment. The author presents the viability of launching an IoT project in a smart village 
with limited upfront investment and minimum external funding. The author considered WiFi for the networking infrastructure as opposed to LPWAN technologies including LoRaWan. To this end, with a single gateway's capability to serve the whole smart village, replacing the WiFi APs with a single LoRa gateway could reduce the number of cellular data subscriptions would increase the cost of hardware equipment. On the other hand, the author acknowledges the popularity, shareability, and stability of WiFi as its strengths for being preferred today. However, as those aspects are forecast to possibly change in the upcoming years, the paper recommends to set a solid ground for the deployment of LPWan technologies to realize IoT support for smart villages.

Smart parking is an important application in urban smart-city services. The authors of Reference [2] present a smart parking system by exploiting the benefits of WSNs. The WSN-based smart parking system calls an adaptable and hybrid self-organization algorithm for the WSN so that it runs under both linear and mass parking cases while providing a better energy-management service for sensors so that the battery lifetime of every sensor can be prolonged, which would consequently prolong the lifetime of the entire WSN. Furthermore, besides the communication- and energy-related issues, the system also facilitates driver assistance through an effective search mechanism for available parking spots in the vicinity.

M2M networks are inseparable components of smart-city communication ecosystems. The authors in Reference [3] present a priority-based M2M overlay network over LTE for smart-city services. Thus, the overlay network is designed to allow the coexistence of a massive number of M2M devices with Human-to-Human $(\mathrm{H} 2 \mathrm{H})$ traffic, and further access the network bypassing the full LTE handshake procedure. In order to support multiple priority classes in the M2M network traffic, the IEEE 802.15.6 standard is adopted. Performance analysis of the M2M system combined with the $\mathrm{H} 2 \mathrm{H}$ traffic reveals the trade-offs required to meet the targets for sufficient performance and reliability for M2M traffic when the $\mathrm{H} 2 \mathrm{H}$ traffic intensity is known upfront. The authors show that their performance results are promising to support this approach in various applications including crowd sensing, smart-city monitoring, and beyond.

Smart cities also involve the introduction of policies for sustainability and community health at the municipal and governmental levels. The authors of Reference [4] present a smart waste-management system that uses WSNs to monitor accumulated waste levels in garbage bins within the borders of a municipal region. The data collected from the WSN are aggregated in a Cloud platform and are fed into a fast heuristic algorithm to determine the number of trucks, route per truck, and the order of collection per bin in order to minimize the delay for the citizens, and/or minimize the cost of garbage collection (in terms of mileage cost and pollution penalties) from the municipality's standpoint. The authors also present optimization models to verify the effectiveness of their proposed heuristic approaches.

Activity recognition aims at effective user-support systems in smart environments. The authors of Reference [5] present a user-support system for fine-grained activity recognition by using two main sources: wearables and cameras. The proposed system aims to identify the text at which a user is gazing. This is based on the assumption that the text content is related to the user activity at that time. The authors point out the fact that the text meaning depends on the location. Thus, they use wearable sensors and a fixed camera so that the global location of the text is acquired via image matching by using the local features of the images captured by these devices. Then, the feature vector is coupled with the content of the text. The authors present experimental results with real participants in a lab environment.

Smart health is an important application area in smart cities, and gait monitoring is one of the fundamental well-being aspects. With this in mind, the authors of Reference [6] present a SmartInsoles Cyber-Physical System (CPS) to measure gait parameters of multiple users in a restriction-free setting. Participants involved in the experimental study performed 10 walks on the Tekscan Strideway gait-mat system, while the SmartInsoles CPS system was worn at the same time. Analysis of spatiotemporal data reveals useful information about seven parameters, namely, stride time, stance time, swing time, double support time, step time, cadence, and gait time. The authors conclude that the outputs of the 
two systems highly coincide, and the presented CPS system offers high accuracy as a multidevice multisensory system for gait monitoring and analysis.

Battery lifetime of wireless sensor nodes has been a challenge since the very first days of WSNs. The ubiquity of smart-city services can only be ensured with the significantly increased lifetime of sensors and WSNs. The authors of Reference [7] propose an efficient solar-energy-harvesting system with pulse-width modulation (PWM) and maximum power-point tracking (MPPT) to sustain the batteries of WSN nodes. Following the design of several models for a solar-energy harvester system, the authors run a series of simulations for solar powered DC-DC converters with PWM and MPPT to obtain optimum results. The simulation study showed that the ambient solar-energy harvesting system could ensure $87 \%$ and $96 \%$ efficiency by using PWM control and MPPT control techniques, respectively. In order to validate their simulations, the authors also present an experimental study for the PWM-controlled solar-energy-harvesting WSN.

In accordance with energy efficiency of WSNs, energy harvesting solutions should also be complemented with smart network protocols. As a protocol-level solution to sustain WSNs in smart cities, the authors of Reference [8] present a new routing WSN scheme in a context where IoT is used in an opportunistic manner with the motivation of reducing the communication overhead in WSN nodes. In the proposed scheme, a WSN is deployed in a smart-city setting, and it forwards the data toward the sink node by interacting with IoT devices. To enable WSN-IoT interaction in an opportunistic manner so as to significantly reduce the energy consumption of the WSN nodes, the authors presented a prototype integration platform. The authors evaluated their proposal in a simulation environment and presented interesting results that support the viability of opportunistic IoT usage in WSN routing.

\section{Conclusions}

The Special Issue on Wireless Sensor and Actuator Networks for Smart Cities features eight high-quality articles, each of which addresses a different aspect of the subject. The contributions to this Special Issue can be classified under two categories: application-driven/application-specific studies and infrastructure/communication-driven studies. The former presents a selection of high-quality works that tackle the effective use of WSANs on smart parking, user-support systems, smart health, and smart waste management from the standpoint of application layer. The latter includes a pool of high-quality studies that aim to address the communication challenges in the deployment of WSANs, their coexistence with other wireless-networking technologies in smart cities, and overcoming battery limitations through the lens of power and communications engineering.

In spite of the variety of their research foci under the scope of this Special Issue, all of the featured articles in this issue are in agreement that research on WSANs for smart cities will continue to uncover many outstanding issues and challenges for researchers and professionals in the sectors that are involved with projects for realizing smart cities and communities.

Acknowledgments: We would like to thank Dharma P. Agrawal (Editor-in-Chief) for his support in guest-editing this Special Issue. We also greatly appreciate the assistance of everyone in the editorial office, and particularly the managing editor, Louise Liu. Furthermore, the anonymous reviewers of this Special Issue have helped us significantly, and they all deserve very special thanks. Last but not least, we would like to thank all authors who have contributed to this Special Issue. Without their contributions, this Special Issue would not have been made possible.

Conflicts of Interest: The authors declare no conflict of interest.

\section{References}

1. Ciuffoletti, A. Low-Cost IoT: A Holistic Approach. J. Sens. Actuator Netw. 2018, 7. [CrossRef]

2. Hilmani, A.; Maizate, A.; Hassouni, L. Designing and Managing a Smart Parking System Using Wireless Sensor Networks. J. Sens. Actuator Netw. 2018, 7. [CrossRef]

3. Khan, N.; Mišić, J.; Mišić, V.B. Priority-Based Machine-To-Machine Overlay Network over LTE for a Smart City. J. Sens. Actuator Netw. 2018, 7. [CrossRef] 
4. Omara, A.; Gulen, D.; Kantarci, B.; Oktug, S.F. Trajectory-Assisted Municipal Agent Mobility: A Sensor-Driven Smart Waste Management System. J. Sens. Actuator Netw. 2018, 7. [CrossRef]

5. Chiba, S.; Miyazaki, T.; Sugaya, Y.; Omachi, S. Activity Recognition Using Gazed Text and Viewpoint Information for User Support Systems. J. Sens. Actuator Netw. 2018, 7. [CrossRef]

6. Arafsha, F.; Hanna, C.; Aboualmagd, A.; Fraser, S.; El Saddik, A. Instrumented Wireless SmartInsole System for Mobile Gait Analysis: A Validation Pilot Study with Tekscan Strideway. J. Sens. Actuator Netw. $2018,7$. [CrossRef]

7. Sharma, H.; Haque, A.; Jaffery, Z.A. Modeling and Optimisation of a Solar Energy Harvesting System for Wireless Sensor Network Nodes. J. Sens. Actuator Netw. 2018, 7. [CrossRef]

8. Hanif, S.; Khedr, A.M.; Al Aghbari, Z.; Agrawal, D.P. Opportunistically Exploiting Internet of Things for Wireless Sensor Network Routing in Smart Cities. J. Sens. Actuator Netw. 2018, 7. [CrossRef]

(C) 2018 by the authors. Licensee MDPI, Basel, Switzerland. This article is an open access article distributed under the terms and conditions of the Creative Commons Attribution (CC BY) license (http:/ / creativecommons.org/licenses/by/4.0/). 